\title{
Cohesive debonding of a stiffener from an elastic
}

\section{substrate}

\author{
Annalisa Franco ${ }^{\mathrm{a}}$, Gianni Royer-Carfagni ${ }^{\mathrm{b}, *}$ \\ ${ }^{a}$ Department of Civil Engineering University of Pisa, Via Diotisalvi 2, I 56126 Pisa, \\ Italy \\ ${ }^{b}$ Department of Industrial Engineering, University of Parma, Parco Area delle Scienze \\ 181/A, I 43100 Parma, Italy
}

\begin{abstract}
To strengthen concrete or masonry, a modern technique uses adherent strips made of Fiber Reinforced Polymer (FRP). A model problem for this is here considered, represented by an elastic stiffener pulled at one end, in adhesive contact with an elastic half space in generalized plane stress. An analytical solution is developed under the hypothesis $\grave{a}$ la Baranblatt that cohesive adhesion forces remain active between the two materials when relative slip occurs (provided this is less than a critical value), so that the stress singularity predicted by the theory of elasticity in the case of perfect bonding is removed. We find that the bond length beyond which no further increase of strength could be achieved, referred to as the effective bond length, coincides in practice with the ultimate length of the cohesive zone, i.e., its maximal extension prior that the critical slip limit is attained. The debonding process in a pull-out experiment is analyzed in detail. Results are in better agreement with experimental data than those obtainable with traditional models, which neglect as a rule the deformation of the substrate.
\end{abstract}

Keywords:

Elastic stiffener, contact, cohesive forces, debonding, Fiber Reinforced Polymer (FRP), Chebyshev polynomials.

*Corresponding Author. Tel.: +39 0521 905896; fax: +39 0521905705

Email address: gianni.royer@unipr.it (Gianni Royer-Carfagni) 


\section{Introduction}

Motivation for this work is a widely-used technique that employs strips/ plates of Fiber Reinforced Polymer (FRP) to strengthen concrete and masonry structures. The performance of the bond between stringers and substrate plays a key role in the effectiveness of the reinforcement, so that a wide research has been focused on the mechanical response of the bonded joints in the load transfer process. Experimental results have provided a wealth of evidence that both in flexural and shear strengthening applications the most frequent failure mode is the debonding of the FRP plate from the substrate, triggered by high stress concentrations at the ends of the stiffener. Shear crack propagation parallel to the FRP-concrete interface eventually leads to the complete separation of the materials, causing the sudden decrease of both structural stiffness and strength.

Although a mixed-mode analysis (Hutchinson and Suo, 1991; Ascione and Mancusi, 2010; Begley et al., 2012; Williams and Hadavinia, 2002) accounting for the normal stresses acting at the interface is certainly the most accurate approach, it has been shown in Freund and Suresh (2008) that the membrane assumption for the stiffener is perfectly acceptable when its thickness is "small" with respect to the characteristic size of the system" ${ }^{1}$. Under this condition, the failure mechanism for a straight ${ }^{2}$ thin strip bonded to an elastic substrate can be considered in pure mode II.

To better understand the phenomenon of mode II fracture propagation in FRP-to-concrete bonded joints, attention has been paid to the analysis of the shear-stress distribution at the interface. Experimental tests have been conducted with different setups, including single shear tests (Taljsten, 1997; Bizindavyi and Neale, 1999; Mazzotti et al., 2008; Carrara et al., 2011; Yao et al., 2005), double shear tests (Maeda et al., 1997; Brosens and Van Gemert, 1997) and modified beam tests (De Lorenzis et al., 2001), for which an extensive list of references can be found in Yao et al. (2005); Chen and

\footnotetext{
${ }^{1}$ Freund et al. (Freund and Suresh, 2008) have given a qualitative indication for the thickness of the stiffener, which has to be at least 20 times smaller than the characteristic size (usually the height of the substrate) to assure a membrane behavior.

${ }^{2}$ Indeed, this is true also when the substrate is moderately curved (Ascione and Mancusi, 2012).
} 
Teng (2001). In general, in pull-out tests the axial force in the stiffener is gradually transmitted to the substrate by shear forces acting at the interface. Such forces decay very quickly passing from the loaded end to the the free end of the stiffener, so that they can be considered active on a certain length only, usually referred to as the effective bond length or the effective stress transfer length. In long stiffeners, as the load increases, debonding near the applied load shifts the stress transfer zone to new areas farther away from the loading point, confirming that only part of the bond is active. In other words, the anchorage strength does not increase with an increase of the bond length beyond its active limit. However, a longer bond length may improve the ductility of the failure process due to the gradual translation of the effective length, as debonding proceeds. This phenomenon has been confirmed by many studies on steel-to-concrete (Taljsten, 1997) and FRPto-concrete bonded joints (Maeda et al., 1997).

To interpret this mechanism, various shear-anchorage-strength models have been proposed, for which a review can be found in Chen and Teng (2001). In general, these models can be classified into three categories: $i$ ) empirical models based on the regression of test results (Maeda et al., 1997); ii) engineering formulations based upon simplified assumptions and appropriate safety factors (Brosens and Van Gemert, 1997; Chen and Teng, 2001; Mancusi and Ascione, 2012); iii) fracture-mechanics-based models (Holzenkämpfer, 1994; Yuan et al., 2001; Neubauer and Rostasy, 1997). Despite the variety of the reinforcing materials, of the strengths of the substrates and of the geometry of the stiffeners, there is a general agreement on many aspects of the failure process. Since it has been experimentally verified that increasing the bond length beyond a certain limit does not lead to any increase of loadcarrying capacity, all models aim at defining such limit, usually referred to as the effective bond length.

It is evident that the determination of the effective bond length is of fundamental importance in the characterization of the joint. To our knowledge, the totality of the analytical anchorage-strength models neglects the elastic deformation of the substrate and assumes a shear vs. slip interface constitutive law to describe the entire phenomenon. Such models predict a fast (usually exponential) decay of the transfer shear stress from the loaded to the free end of the stringer that never reaches the zero value, whatever the length of the stiffener is. Since no part of the stiffener is inactive regardless of its 
length, the definition itself of effective bond length needs an engineering interpretation. For example, many researchers define the effective bond length as the bond length over which the shear stresses offer a total resistance which is at least $97 \%$ of the ultimate load $^{3}$ of an infinite joint (Yuan et al., 2001, 2004; Wu et al., 2002; Chen et al., 2012). According to other authors, the evaluation cannot but be purely experimental. Measuring the strain profile in the stiffener - usually employing resistance strain gages - the effective bond length is the length over which the strain decays from the maximum to the zero value (Ali-Ahmad et al., 2006; Bizindavyi and Neale, 1999; Carloni and Subramaniam, 2010; Carloni et al., 2012; Kamel et al., 2004; Subramaniam et al., 2007).

There are some intrinsic ambiguities related to these definitions. In the first case, the definition is associated with an a priori defined percentage of load, and the result strongly depends upon the model employed, i.e., the particular bond-slip constitutive law. The second definition cannot get rid of the experimental approximations and depends upon the sensitivity of the gages. Moreover, it implicitly assumes that the deformation of the substrate is negligible. Indeed, this is a general hypothesis supported by the greatest majority of authors (see also Carrara et al. (2011); Ferracuti et al. (2007); Mazzotti et al. (2008)), because it gives substantial simplification in the modelling. Consequently the slip, i.e., the relative displacement between stiffener and substrate, is evaluated by simply integrating the axial strain in the stiffener. In any case, the hypothesis of a rigid substrate implies that the slip is always nonzero whatever the bond length is.

The present article considers the effect of the substrate elasticity, usually neglected, by analyzing the contact of a finite elastic stiffener bonded to the boundary of a semi-infinite plate, supposed in generalized plane stress. This kind of problems in plane linear elasticity has been considered by different authors (Arutiunian, 1968; Benscoter, 1949; Brown, 1957; Koiter, 1955; Melan, 1932; Reissner, 1940), with the main purpose of evaluating the stress concentrations near the edges of the stiffener in relation with crack initiation and propagation in the substrate or along the interface. More recent studies include the case of a rigid line inclusion embedded in an infinite prestressed

\footnotetext{
${ }^{3}$ Notice that $\tanh 2 \simeq 0.97$ : this is a characteristic value in the solution of the differential equations governing the debonding process (Yuan et al., 2001).
} 
substrate (Dal Corso et al., 2008), to which a generic perturbation field is superimposed, as well as the case of reinforced no-tension-materials (De Faveri et al., 2012).

Following this rationale, in previous work (Franco and Royer-Carfagni, 2012) the authors have considered the case of a perfectly-adherent stiffener, focusing the attention on the debonding process assumed to begin, and continue, as soon as the energy release rate due to an infinitesimal delamination becomes equal to the interfacial fracture energy (Griffith balance). The main drawback of this approach was the difficulty to give a consistent definition of the effective anchorage length. In fact, when slip is not contemplated, the presence of the stress singularities at both ends of the stiffener produces a very rapid decay of the shear stress profile at the interface, which does not agree with experiments.

This work aims at solving this inconsistency by introducing a cohesive zone where slippage can occur. Following the approach originally proposed by Barenblatt (Barenblatt, 1962), as suggested by other authors (Chen et al., 2009; Shield and Kim, 1992) for similar-in-type problems, the length of the cohesive zone for a fixed load is evaluated by imposing that the stress intensity factor at the end of the bonded zone is null, eliminating the singularities which are predicted by the theory of elasticity. Effective material separation is supposed to start when the relative slip exceeds a certain threshold. If the stiffener is sufficiently long, there is maximal reachable length of the cohesive zone; in a strain-driven pull out test, the cohesive portion simply translates along the stiffener as debonding proceeds, maintaining its length unchanged, while the load remains almost constant. A strain softening phase, usually associated with snap-back, is entered when the cohesive zone reaches the free end of the stiffener.

We will demonstrate that there is a natural definition of effective bond length, associated with the maximal length of the cohesive zone reached in sufficiently long stiffeners. The model is able to interpret the debonding process step-by-step, evidencing different-in-type responses when the bond length is higher or lower than the effective bond length. The response is characterized in terms of load-displacement curves, furnishing values in good agreement with experimental data drawn by the technical literature. 


\section{The model problem: cohesive adhesion of a stiffener to a semi- infinite plate.}

As shown in Figure 1, consider an elastic stiffener of length $l$, thickness $t_{s}$ and constant width $b_{s}$, bonded to the an elastic semi-infinite plate in generalized plane stress. At one end, the stiffener is loaded by a longitudinal concentrated force $P$. If no slippage occurs between stiffener and plate, the theory of elasticity predicts that interface shear forces have a singularity at both ends of the stiffener (Franco and Royer-Carfagni, 2012).

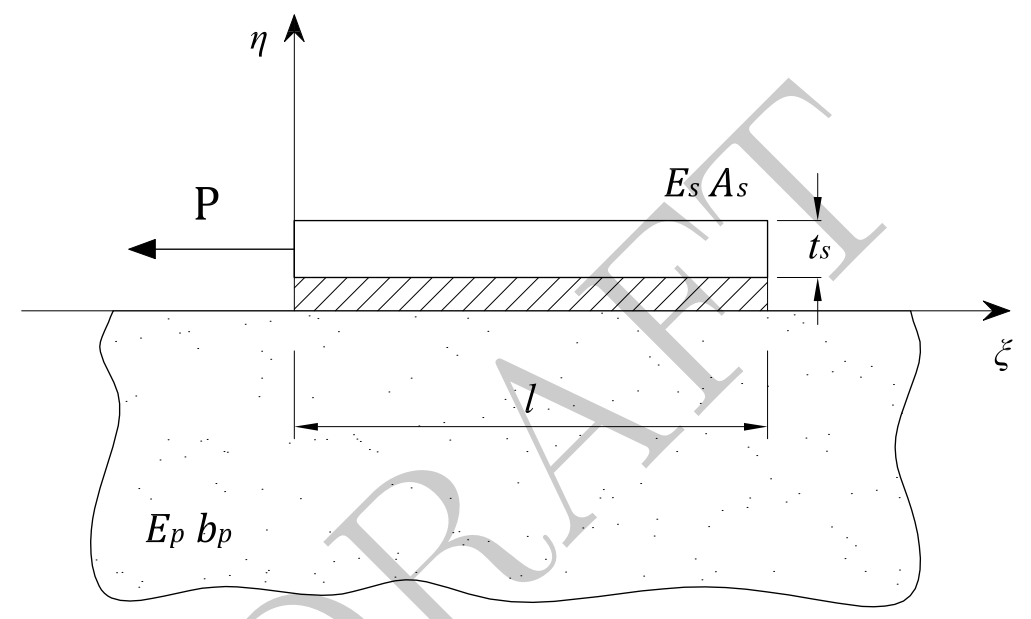

Figure 1: A finite stiffener bonded to the boundary of a semi-infinite plate and pulled at one end.

To remove this physically-inconsistent singularity, a cohesive zone is now introduced, where slippage can take place between the two parts. This is a free boundary problem, because the length of this zone is unknown and must be found from condition that interface forces are finite. Indeed, this is the same rationale followed by Barenblatt in the theory of cohesive cracks (Barenblatt, 1962).

As indicated in Figure 2, let $c$ denote the length of the cohesive zone. A reference system $(\xi, \eta)$ is introduced with the origin on the left-hand-side edge of the stringer, so that the cohesive zone is $0 \leq \xi \leq c$, while the completely bonded part lays in the interval $c \leq \xi \leq l$.

Referring to the free-body diagram of Figure 3, equilibrium of that part 


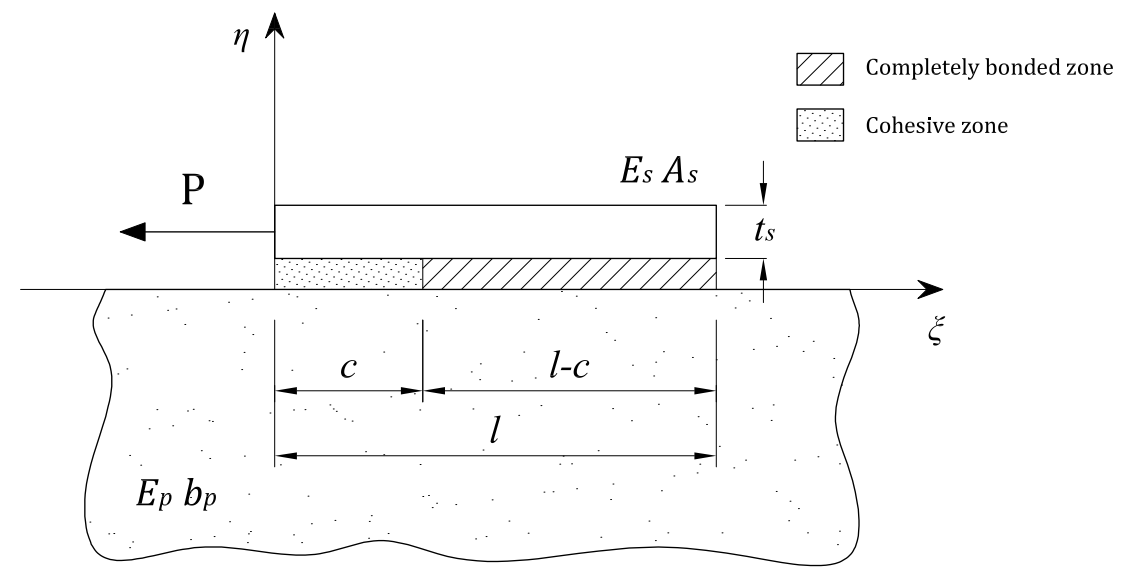

Figure 2: A finite stiffener bonded to the boundary of a semi-infinite plate with a cohesive zone.

of the stiffener comprised between the origin and a section $\xi=x$ allows to write the axial force $N_{s}(x)$ in the form

$$
N_{s}(x)=P-\int_{0}^{c} q_{c}(\xi) d \xi-\int_{c}^{x} q(\xi) d \xi
$$

where $q_{c}(\xi)$ is the (cohesive) tangential force per unit length acting over the length $c$, while $q(\xi)$ is the contact tangential force per unit length in the bonded portion.

By Hooke's law, the stiffener strain reads

$$
\varepsilon_{s}(x)=\frac{N_{s}(x)}{E_{s} A_{s}}=\frac{1}{E_{s} A_{s}}\left[P-\int_{0}^{c} q_{c}(\xi) d \xi-\int_{c}^{x} q(\xi) d \xi\right]
$$

where $E_{s}$ is its elastic modulus and $A_{s}$ its cross sectional area. Besides, on the boundary of the semi-infinite plate, the strain in the interval $[0, l]$ due to the cohesive stress and to the tangential contact stress may be written in the form (Grigolyuk and Tolkachev, 1987)

$$
\varepsilon_{p}(x)=-\frac{2}{\pi E_{p} b_{p}}\left[\int_{0}^{c} \frac{q_{c}(\xi)}{\xi-x} d \xi+\int_{c}^{l} \frac{q(\xi)}{\xi-x} d \xi\right]
$$




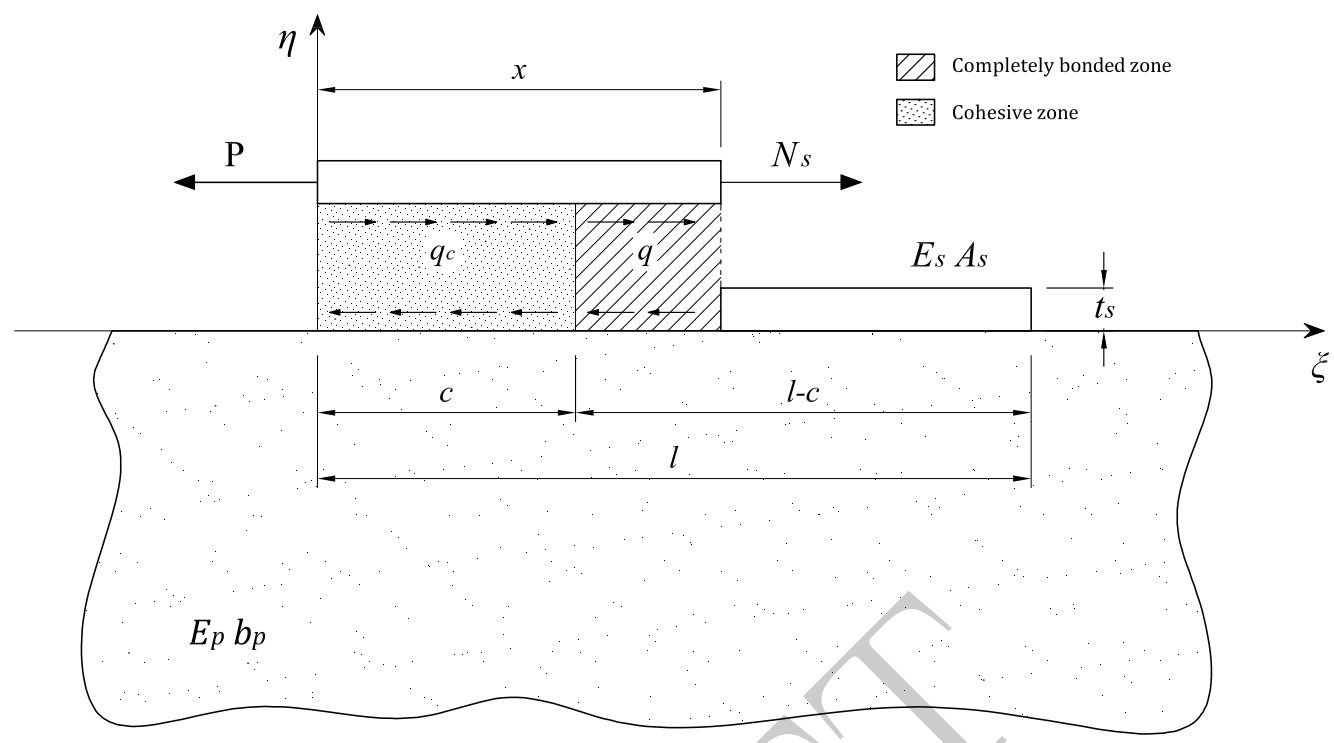

Figure 3: A finite stiffener bonded to the boundary of a semi-infinite plate. Free body diagram of a portion of the stiffener.

where $E_{p}$ is the elastic modulus of the plate and $b_{p}$ its thickness. One obtains the singular integro-differential equation that solves the problem by imposing that strains are equal over the interval of contact.

In the simplest case one may assume that the cohesive forces are constant, i.e., $q_{c}(\xi)=$ const. $=q_{c}$. Consequently, by equating (2.2) and (2.3), one finds

$\frac{1}{E_{s} A_{s}}\left[\left(P-q_{c} c\right)-\int_{c}^{x} q(\xi) d \xi\right]=-\frac{2}{\pi E_{p} b_{p}}\left[q_{c} \ln \left|\frac{x-c}{x}\right|+\int_{c}^{l} \frac{q(\xi)}{\xi-x} d \xi\right], \forall x \in(c, l)$,

whose solution is subjected to the equilibrium condition for the stiffener

$$
\int_{0}^{c} q_{c} d \xi+\int_{c}^{l} q(\xi) d \xi=P
$$

A change of variable is convenient, so that the completely bonded zone would lay in the interval $[-1,1]$. This is done provided that 


$$
t=2 \frac{(\xi-c)}{(l-c)}-1 \Longleftrightarrow \xi=\frac{(l-c)}{2}(t+1)+c
$$

In the following, $t$ will always denote the variable associated with the interval $[-1,1]$, representing the image of the bonded length through a mapping of the type (2.6). If $f(\xi)$ is a generic function of the variable $\xi$, a new function, say $\bar{f}(t)$, should be defined such that $\bar{f}(t)=f(\xi(t))$ when (2.6) is applied. However, with a little abuse of notation but without risk of confusion, we will drop this distinction and simply write $f(\xi)=f(t)$, with the understanding that $f(t)=f(\xi(t))$.

Therefore, introducing the rigidity parameter $\lambda$, defined as

$$
\lambda=\frac{2}{\pi} \frac{E_{p} b_{p}(l-c)}{E_{s} A_{s}}
$$

equation (2.4) can be written in the form

$$
q_{c} \ln \left|\frac{(l-c)\left(t_{0}+1\right)}{(l-c) t_{0}+(l+c)}\right|+\int_{-1}^{1} \frac{q(t)}{t-t_{0}} d t=-\frac{\pi^{2} \lambda}{8}\left[\frac{2\left(P-q_{c} c\right)}{l-c}-\int_{-1}^{t_{0}} q(t) d t\right],
$$

while the equilibrium condition (2.5) becomes

$$
\int_{-1}^{1} q(t) d t=\frac{2\left(P-q_{c} c\right)}{l-c}
$$

Solution for (2.8) can be obtained by expanding the contact force $q$ in term of a series of Chebyshev polynomials ${ }^{4}$ (Grigolyuk and Tolkachev, 1987), which are orthogonal in the interval $[-1,1]$, in the form

\footnotetext{
${ }^{4}$ For the sake of completeness, the main properties of Chebyshev polynomials are recalled in the Appendix.
} 


$$
q(t)=\frac{2\left(P-q_{c} c\right)}{\pi(l-c) \sqrt{1-t^{2}}} \sum_{s=0}^{n} X_{s} T_{s}(t)
$$

where $X_{s}$ are constants to be determined. Observe that there is a square-root singularity in the solution at both ends of the reinforcement, which is typical of most contact problems in linear elasticity theory. Following Bubnov's method (Grigolyuk and Tolkachev, 1987), with a procedure similar to that of Franco and Royer-Carfagni (2012), substitution of (2.10) into conditions (2.9) and (2.8) allows to obtain, with the orthogonality conditions for Chebyshev polynomials of the first kind (see Appendix),

$$
X_{0}=1
$$

and the system of linear equations

$$
X_{j}+\frac{\lambda}{4} \sum_{s=1}^{n} a_{j s} X_{s}=-\frac{\lambda}{4} b_{j}-\frac{q_{c}(l-c)}{\pi\left(P-q_{c} c\right)}\left[c_{j}-d_{j}\right], \quad \text { for } j=1,2, \ldots, n
$$

Here

$$
\begin{aligned}
a_{j s} & =1 / s \int_{-1}^{1} U_{j-1}(t) U_{s-1}(t)\left(1-t^{2}\right) d t, \\
b_{j} & =\int_{-1}^{1} U_{j-1}(t) \sqrt{1-t^{2}} \arccos t d t \\
c_{j} & =\int_{-1}^{1} U_{j-1}(t) \sqrt{1-t^{2}} \ln |(l-c)(t+1)| d t \\
d_{j} & =\int_{-1}^{1} U_{j-1}(t) \sqrt{1-t^{2}} \ln |[(l-c) t+(l+c)]| d t
\end{aligned}
$$

being $U_{j}(t)$ the Chebyshev polynomials of the second kind. These expressions can be evaluated with the change of variable $t=\cos \varphi$, so that $U_{j-1}(t(\varphi))=$ $\sin j \varphi / \sin \varphi$. In conclusion, one finds 


$$
\begin{aligned}
& \begin{cases}a_{j s}=-\frac{4 j}{\left[(j+s)^{2}-1\right]\left[(j-s)^{2}-1\right]}, & \text { for even } j-s, \\
a_{j s}=0, & \text { for odd } j-s,\end{cases}
\end{aligned}
$$

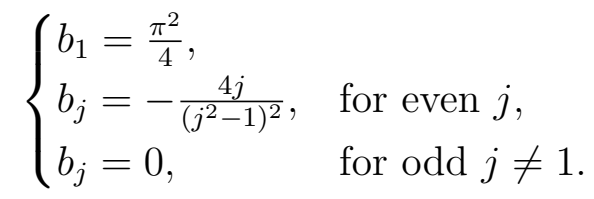

$$
\begin{aligned}
& \begin{cases}c_{1}=\frac{\pi}{4}\left[1+\ln \left|\left((l-c)^{2} / 4\right)\right|\right], & \\
c_{j}=(-1)^{j} \frac{\pi}{\left(j^{2}-1\right)}, & \text { for } j=2,3, . ., n,\end{cases}
\end{aligned}
$$

and

$$
\begin{cases}d_{1}=\frac{\pi}{4 a^{2}}\left[2 b^{2}-2 b \sqrt{b^{2}-a^{2}}-a^{2}+2 a^{2} \ln \left(b+\sqrt{b^{2}-a^{2}}\right)-2 a^{2} \ln 2\right] & \\ d_{j}=\frac{\pi}{2}\left[\frac{1}{j+1}\left(\frac{\left(-b+\sqrt{b^{2}-a^{2}}\right)}{a}\right)^{j+1}-\frac{1}{j-1}\left(\frac{\left(-b+\sqrt{b^{2}-a^{2}}\right)}{a}\right)^{j-1}\right], & \text { for } j=2,3, . ., n,\end{cases}
$$

having posed $a=l-c$ and $b=l+c$.

Notice that the coefficients of the system (2.11) coincide with those derived in Franco and Royer-Carfagni (2012) when the stiffener is completely bonded to the substrate and the cohesive zone is absent. The main difference here is in the constant terms on the right-hand side of (2.11), which depend in particular upon the length $c$ of the cohesive zone. The parameter $c$ adds to the other $n$ unknowns $X_{s}$, so that there are $n+1$ unknowns for the $n$ equations (2.11). Another condition needs to be introduced, and this is accomplished by imposing that in $\xi=c(t=-1)$ the shear stress must be finite, or, equivalently, that the mode II stress intensity factor $K_{I I}$ is null, that is

$$
K_{I I}=\lim _{\xi \rightarrow c} q(\xi) \sqrt{2 \pi(\xi-c)}=0
$$

Substitution of the contact stress (2.10) into (2.12) gives the expression 


$$
K_{I I}=\frac{2\left(P-q_{c} c\right)}{\sqrt{2 \pi(l-c)}} \sum_{s=0}^{n} X_{s}(-1)^{s}
$$

which reduces to the condition

$$
\sum_{s=0}^{n} X_{s}(-1)^{s}=0
$$

under the condition that, of course, $(l-c)>0$. This is the adaptation to the contact problem of the approach originally proposed by Barenblatt (Barenblatt, 1962) to eliminate the stress singularity predicted by the elasticity theory in an opening crack, as a consequence of cohesive forces acting at its tip. Condition (2.14) allows to evaluate the length of a zone over which tangential slippage can occur at the interface, provided that the cohesive stress $q_{c}$ is known.

\section{Analysis of the debonding process. The case of FRP reinforce- ment of concrete}

The coefficients of the system (2.11) and condition (2.14) depend upon the elastic properties of the materials in contact. Once they have been defined, the model should predict the critical load and the effective anchorage length of the stiffener in order to assure a reliable reinforcement avoiding delamination. Moreover, it is also possible to analyze the debonding process that occurs at the interface between substrate and stiffener, as the latter is gradually pulled. The proposed model is now applied to the particular case of a Fiber-Reinforced-Polymer (FRP) stringer bonded to a concrete substrate. Other types of materials can be treated with the same procedure.

\subsection{Shear-stress vs. slip constitutive relationship at the interface}

In most technical standards, the typical bond-stress vs. slip $(\tau-s)$ relationship for a FRP/concrete interface is approximated by a trilateral of the type shown in Figure 4, with a linearly ascending branch till the peak stress 


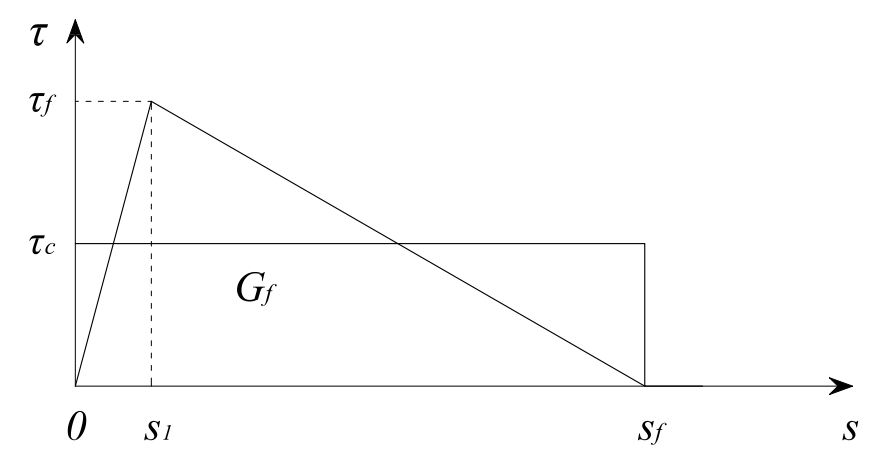

Figure 4: Shear-stress vs. slip constitutive relationship at the interface.

$\tau_{f}$, followed by a linear softening phase until the shear stress reduces to zero $\left(\tau=0\right.$ and $\left.s=s_{f}\right)$, and a final zero-stress plateau.

On the other hand, equations (2.11) have been derived under the hypothesis that the cohesive force per-unit-length $q_{c}$ is constant on the slip zone. Assuming that stress is constant on the width $b_{s}$ of the interface, this implies that the the shear bond stress equals $\tau_{c}=q_{c} / b_{s}$. Consideration of a trilinear constitutive relationship would lead to tremendous complications and this is why an equivalence is sought that maintains the same delamination fracture energy $G_{f}$ and the same slip limit $s_{f}$. This is achieved, as represented in Figure 4 , when the value of the tangential stress $\tau_{c}$ is set equal to half the peak stress $\tau_{f}$ of the trilinear model. Representative values are reported later on in Table 2 with reference to experimental measurements (Ali-Ahmad et al., 2006; Carrara et al., 2011; Mazzotti et al., 2008; Yuan et al., 2004).

As highlighted in various experimental and numerical works (Ali-Ahmad et al., 2006; Carrara et al., 2011), the force vs. displacement response of a bonded joint strongly depends upon the bond length $l$. "Short" stiffeners show a post-peak softening while "long" stiffeners are characterized by a plateau, usually followed by a snapback phase (Figure 5). These two cases need to be distinguished in the analytical interpretation.

In the model of Section 2, relative slip takes place in the cohesive zone, whereas adhesion is perfect on the remaining part of the bond length. For any given pull out force $P$ it is possible to calculate the length $c$ of the cohesive zone for which the cohesive force per unit length $q_{c}$, supposed uni- 

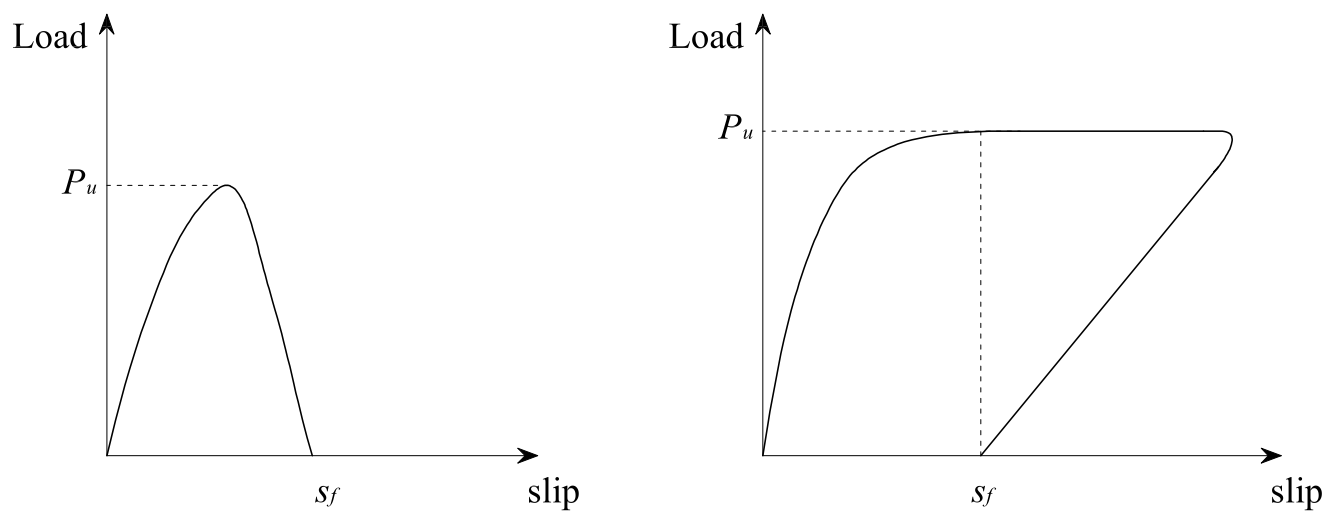

Short stiffeners

Long stiffeners

Figure 5: Typical experimental load-slip curves for a FRP-to-concrete bonded joint.

formly distributed, can annihilate the stress singularity at the extremity of the adherent part. From the elastic solution, it is also possible to calculate the relative slip between stiffener and substrate; debonding starts when the relative slip reaches the limit value $s_{f}$ (Figure 4). The overall response will be different in type in the case of "long" stiffeners and "short" stiffeners.

\subsection{Load-displacement curve for long stiffeners.}

Suppose that in the undistorted reference configuration the stringer is bonded over its length $l$ (Figure 1). Then, the load $P$ is gradually applied at the left hand side. We consider an hypothetical strain-driven test where we control the relative displacement of the loaded end of the stiffener with respect to the substrate, until debonding starts. From that point on, equilibrium configurations are sought as the length of the debonded zone increases.

The typical load-slip curve for this case is shown on the right hand side of Figure 6 . The curve can be characterized by three branches, which represent respectively the strain-hardening, plateau and strain-softening phases. The graph is marked by a series of key-points that correspond to step changes in the response.

The relative slip between stiffener and plate needs to be established at 
(A)

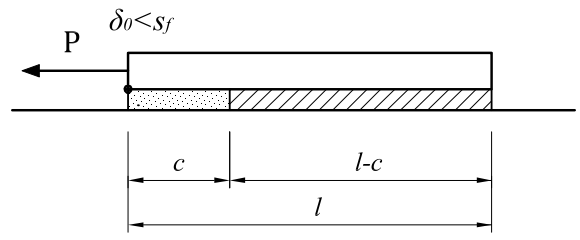

(B)

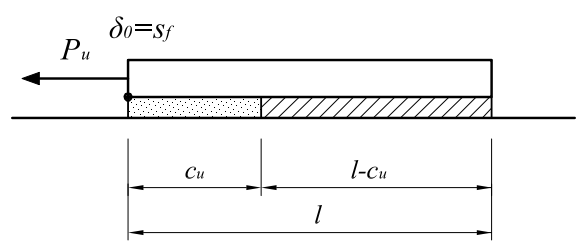

(C)

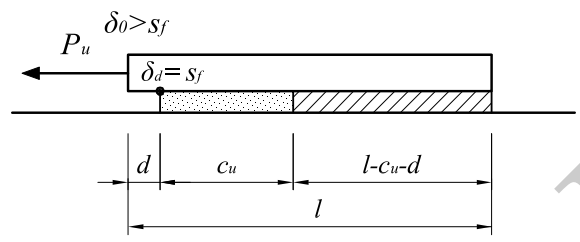

(D)

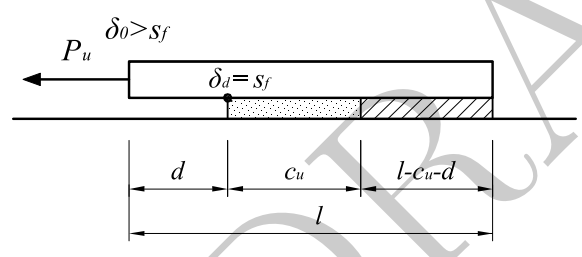

(E)

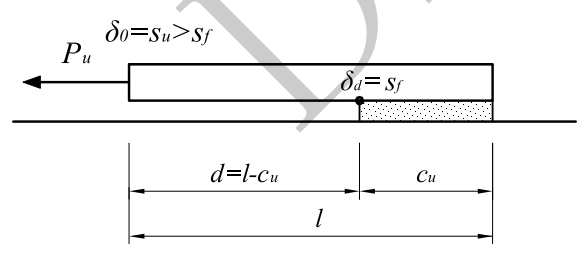

(F)
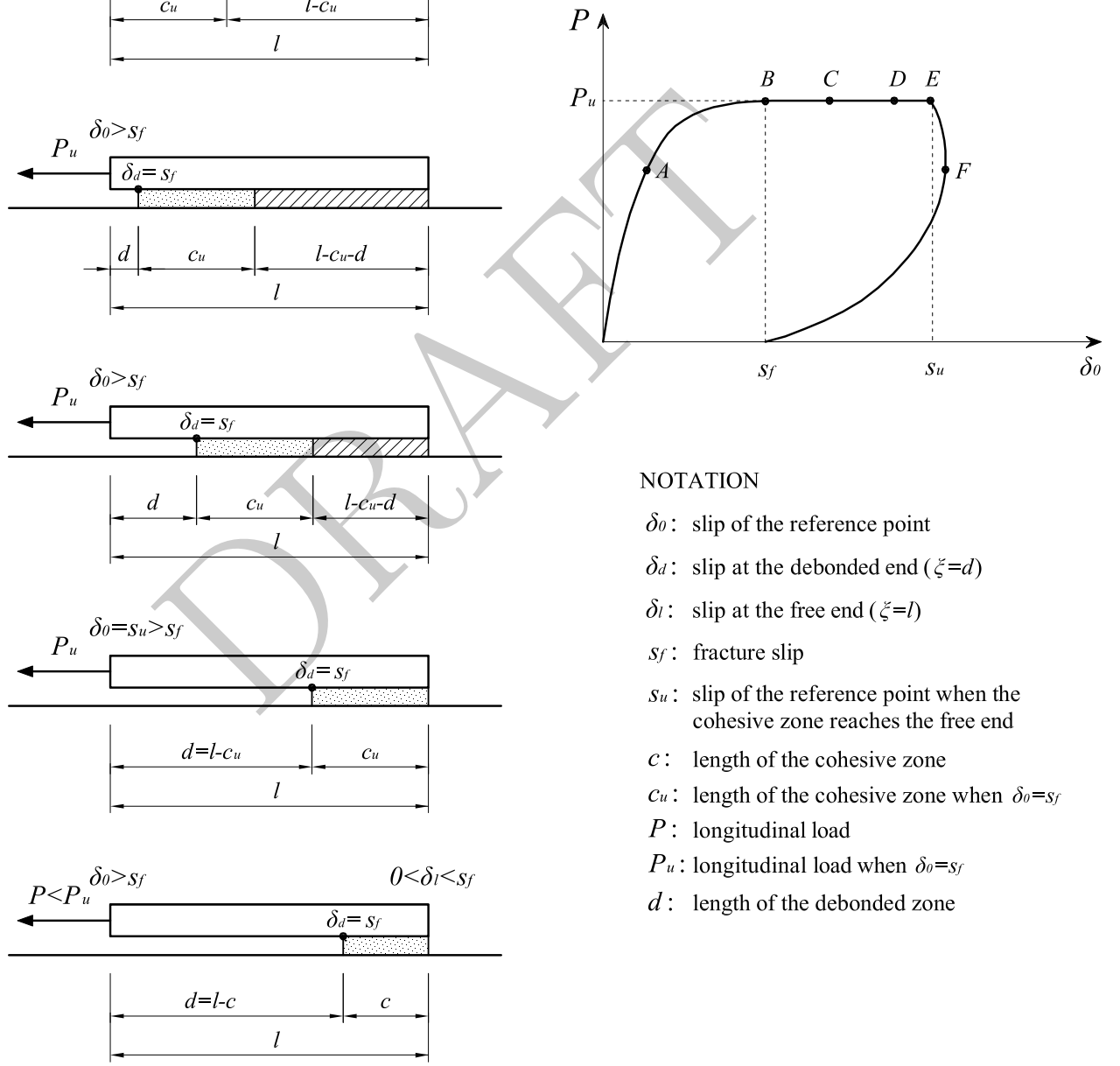

\section{NOTATION}

$\delta_{0}:$ slip of the reference point

$\delta_{d}:$ slip at the debonded end $(\xi=d)$

$\delta_{l}:$ slip at the free end $(\xi=l)$

$s_{f}:$ fracture slip

$S_{u}$ : slip of the reference point when the cohesive zone reaches the free end

$c$ : length of the cohesive zone

$c_{u}$ : length of the cohesive zone when $\delta_{0}=S_{f}$

$P$ : longitudinal load

$P_{u}$ : longitudinal load when $\delta_{0}=S_{f}$

$d$ : length of the debonded zone

Figure 6: Response for long stiffeners. A) development of the cohesive zone; B) initiation of debonding at the loaded end $\left(\delta_{0}=s_{f}\right)$; C)-D) propagation of debonded zone; E) the cohesive zone reaches the free end; F) strain softening branch. 
various points. Hereinafter, the slip at $\xi$ may be denoted with $\delta(\xi)$, but to simplify we will we use the concise notation

$$
\left.\delta(\xi)\right|_{\xi=\xi_{0}} \equiv \delta_{\xi_{0}}
$$

In general, the reference point for the force vs. slip graphs will be the loaded end $\xi=0$ (Figure 2); here the slip, according to (3.1), will be referred to as $\delta_{0}$.

i) Strain hardening branch (point A).

The first, strain hardening phase, marks the development of the cohesive zone. Using equations (2.11) with condition (2.14), each value of the load $P$ is associated with a unique value of the cohesive length $c$. Such equations are non linear in $c$, so that a root-finding algorithm has to be used. Once $P$ and the corresponding $c$ are known, the value of the slip $\delta_{0}$ at the loaded end $\xi=0$ can be calculated as

$$
\delta_{0}=u_{s}(0)-u_{p}(0)
$$

where (Figure 2) $u_{s}$ and $u_{p}$ are the displacements of the stiffener and of the plate substrate, respectively, taken positive if leftwards, i.e., in opposite direction of the $\xi$ axis. Then, for the situation sketched in Figure 6 (A), the relative displacement of a point $x$ comprised in the interval $[0, c]$ can be written as (recall the positive verse of displacements)

$$
\begin{aligned}
& u_{s}(x)-u_{s}(c)=\int_{x}^{c} \varepsilon_{s}(\xi) d \xi=\frac{1}{E_{s} A_{s}}\left[P c-q_{c} c^{2} / 2\right] \\
& u_{p}(x)-u_{p}(c)=\int_{x}^{c} \varepsilon_{p}(\xi) d \xi=u_{p}\left(t_{0}\right)-u_{p}(-1)=\int_{t_{0}}^{-1} \varepsilon_{p}(t) \frac{l-c}{2} d t(3)
\end{aligned}
$$

where, in (3.4), we have used the change of variables (2.6). Observing that at $\xi=c$ the plate and stiffener are perfectly bonded so that $u_{s}(c)=u_{p}(c)$, the slip $\delta_{0}$ can thus be calculated from the difference of the terms on the right 
hand side of (3.3) and (3.4). Referring to the expression (2.3) for the plate strain, equation (3.4), evaluated at $x=0\left(t_{0}=-(l+c) /(l-c)\right)$, becomes $^{5}$

$$
\begin{aligned}
& u_{p}\left(t_{0}\right)-u_{p}(-1)= \\
& -\frac{2}{\pi E_{p} b_{p}}\left(P-q_{c} c\right)\left[X_{0} \ln \left(-t_{0}+\sqrt{t_{0}^{2}-1}\right)+\sum_{s=1}^{n} \frac{X_{s}}{s}\left[(-1)^{s}-\left(t_{0}+\sqrt{t_{0}^{2}-1}\right)^{s}\right]\right] .
\end{aligned}
$$

The load $P$ continues to increase as the length $c$ of the cohesive zone increases. The debonding process does not start until the slip $\delta_{0}$ reaches the limit value $s_{f}$ (Figure 6 , point B), i.e., the value of the slip after which the shear stress reduces to zero (Figure 4). At this point the maximum load $P_{u}$ that can be carried by the FRP stringer is attained and correspondingly, the cohesive zone reaches the length $c_{u}$.

ii) Plateau (points B-E).

If the test is strain driven, after point $\mathrm{B}$, debonding propagates along the interface (Figure 6, points C and D). Let $d$ denote the length of the debonded part. Given $d$, one can again calculate with the same procedure just outlined the length $c$ of the cohesive zone and the corresponding value of equilibrium load $P$ that annihilates the stress singularity at $\xi=d+c$. The condition in this case is that, at the point $\xi=d$, the slip $\delta_{d}$ equals the fracture slip $s_{f}$. Remarkably, one finds that $P \simeq P_{u}$ and $c \simeq c_{u}$. In other words, the cohesive zone, once established, remains constant in practice, and moves towards the free end of the bonded joint, leaving the load $P_{u}$ unchanged.

As recalled in the Introduction, the effective bond length is usually defined as that bond length beyond which there is no further increase of the strength of the joint. From the former analysis, it is possible now to identify the length $c_{u}$ as the effective bond length. In fact, the debonding process occurs at constant ultimate load in the way just outlined whatever the bond length is, provided this is higher than $c_{u}$. Indeed, the shear stress in the zone that remains completely bonded decays very fast, so that the entire load $P_{u}$ is

\footnotetext{
${ }^{5}$ Note that the point $t_{0}$ is external to the interval of contact, i.e. $t_{0}<-1$, so integration has to be performed using relation (A.6) for the case $\left|t_{0}\right|>1$.
} 
in practice equilibrated by the shear interface-stress acting in the cohesive portion. The part of the load that is equilibrated by the contact forces in the perfectly bonded region is negligible (here, less than 1\%) and acts in any case in a very small (right) neighborhood of $\xi=d+c$. As a result, we can consequently define "long" stiffeners those for which the bond length is higher than $c_{u}$, which permits the development of the entire stress-transfer zone.

Of course, the displacement $\delta_{0}$ of the reference point $\xi=0$ increases mainly as a consequence of the strain of the debonded portion of the stringer, not any more constrained by the substrate. This is why this phase is associated with a plateau in the $P-\delta_{0}$ graph. In this situation, similarly to (3.5), the displacement of the plate at $\xi=0\left(t_{0}=-\left(l+c_{u}+d\right) /\left(l-c_{u}-d\right)\right)$ can be found from an expression of the form

$$
\begin{aligned}
u_{p}\left(t_{0}\right) & -u_{p}(-1)=-\frac{2}{\pi E_{p} b_{p}}\left\{q_{c}\left(-c_{u} \ln c_{u}+\left(c_{u}+d\right) \ln \left(c_{u}+d\right)-d \ln d\right)\right. \\
& \left.+\left(P-q_{c} c_{u}\right)\left[X_{0} \ln \left(-t_{0}+\sqrt{t_{0}^{2}-1}\right)+\sum_{s=1}^{n} \frac{X_{s}}{s}\left[(-1)^{s}-\left(t_{0}+\sqrt{t_{0}^{2}-1}\right)^{s}\right]\right]\right\} .
\end{aligned}
$$

Observing again that $u_{s}(-1)=u_{p}(-1)$, the slip $\delta_{0}$ at the reference point becomes

$\delta_{0}=\left[u_{s}\left(t_{0}\right)-u_{s}(-1)\right]-\left[u_{p}\left(t_{0}\right)-u_{p}(-1)\right]=\frac{1}{E_{s} A_{s}}\left[P_{u}\left(d+c_{u}\right)-q_{c} c_{u}^{2} / 2\right]-\left[u_{p}\left(t_{0}\right)-u_{p}(-1)\right]$

iii) Strain softening branch (point F).

When the cohesive zone reaches the free end, the strain softening branch is attained (Figure 6, point E). From now on, the interface is purely cohesive and the shear forces are equal to $q_{c}$. If the stringer is pulled further, the relative slip increases and debonding proceeds where the relative slip exceeds the limit value $s_{f}$ of Figure 4. However, this phenomenon is associated with a sudden decrease of the load carrying capacity and the consequent release 
of the stiffener produces in general a snap-back response (Figure 6, point F). This cannot be revealed if the test is strain driven; therefore at this point a new control variable must be introduced. In particular, as done in the experimental tests of Carrara et al. (2011), the control variable can be chosen to be the slip $\delta_{l}$ of the free end $\xi=l$ of the stiffener.

The relative slip $\delta_{0}$ at the reference point $\xi=0$ is then equal to

$$
\delta_{0}=\left[u_{s}(0)-u_{s}(l)\right]-\left[u_{p}(0)-u_{p}(l)\right]+\delta_{l},
$$

where

$$
u_{s}(0)-u_{s}(l)=\frac{q_{c} c}{E_{s} A_{s}}(l-c / 2)
$$

and

$$
u_{p}(0)-u_{p}(l)=-\frac{2}{\pi E_{p} b_{p}}\left[q_{c}(-c \ln c+l \ln l-(l-c) \ln (l-c))\right] .
$$

For any given value of $\delta_{l}$ the corresponding $c$ is found from condition that at $\xi=d \equiv l-c$ the relative slip $\delta_{d}$ equals the limit value $s_{f}$ of Figure 4 . The resulting $P-\delta_{0}$ graph exhibits a snap-back response. If one neglects the strain in the plate and the consequent displacement given by (3.10), the snap back branch exhibits a parabolic trend. As $P \rightarrow 0$, the slip $\delta_{0}$ of the reference point $\xi=0$ tends to the value $s_{f}$.

\subsection{Load-displacement curve for short stiffeners.}

Having defined in section 3.2 the length $c_{u}$ as the effective bond length, we can consequently call "short" stiffeners those for which $l<c_{u}$. The debonding process for this case is sketched in Figure 7.

i) Strain hardening branch (point A).

The first stage is characterized by a strain-hardening branch where the cohesive zone develops. The equilibrium configuration at point $\mathrm{A}$ can be 
(A)

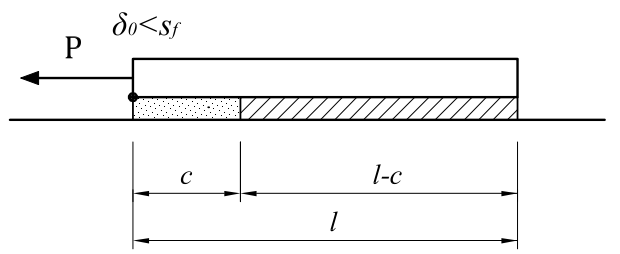

$\square$ Completely bonded zone
Cohesive zone

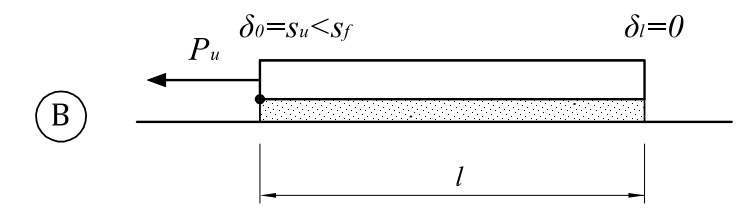

(C)

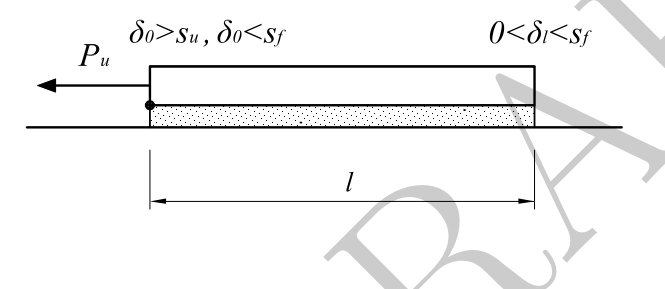

(D)

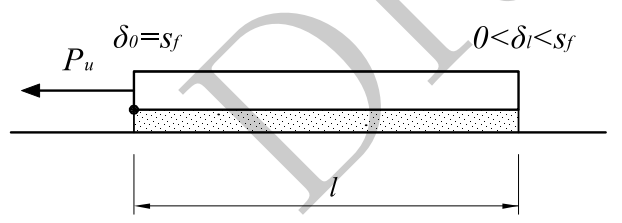

(E)
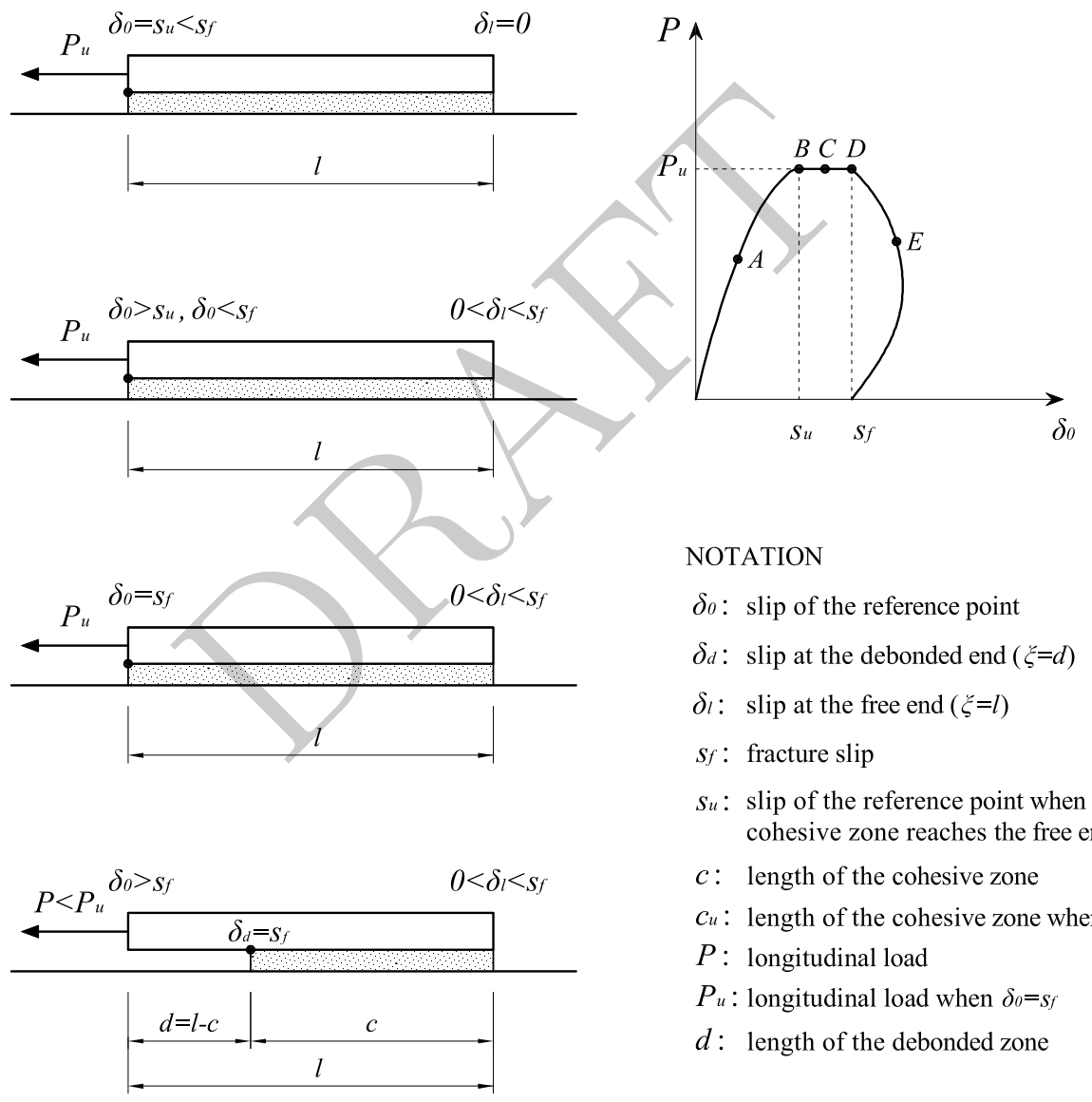

\section{NOTATION}

$\delta_{0}$ : slip of the reference point

$\delta_{d}$ : slip at the debonded end $(\xi=d)$

$\delta_{l}:$ slip at the free end $(\xi=l)$

$S_{f}:$ fracture slip

$s_{u}$ : slip of the reference point when the cohesive zone reaches the free end

$c$ : length of the cohesive zone

$c_{u}$ : length of the cohesive zone when $\delta_{0}=S_{f}$

$P$ : longitudinal load

$P_{u}$ : longitudinal load when $\delta_{0}=s_{f}$

$d$ : length of the debonded zone

Figure 7: Response for short stiffeners. A) development of the cohesive zone; B) the cohesive zone reaches the free end; C) plateau due to rigid slip; D) initiation of debonding $\left(\delta_{0}=s_{f}\right) ;$ E) strain softening branch. 
calculated with the same procedure of Section 3.2. However, now the stringer is too short to permit the development of the entire effective bond length $c_{u}$. Consequently, point B of Figure 7 is characterized by a full cohesive interface with $q=q_{c}$, and a relative slip $\delta_{0}$ of the reference point $\xi=0$ such that $\delta_{0}<s_{f}$. The ultimate load is consequently attained at $P_{u}=q_{c} l$.

ii) Plateau (points $B-D)$.

Augmenting the pull out displacement, the relative slip increases due to a rigid translation of the stringer, characterized by the relative slip $\delta_{l}$ of the free end $\xi=l$. The scenario is that of point $\mathrm{C}$, with the load remaining constantly to $P_{u}$.

It must be clearly remarked that the plateau attained in this case of short stiffeners is different in type from that developing in long stiffeners, discussed in the preceding section 3.2. In short stiffeners the plateau is due to a uniform slip of the completely yielded interface and, consequently, its width can never exceed the limit value $s_{f}$ defined in the constitutive relation of Figure 4. On the other hand, in long stiffeners the plateau is consequent to a progressive translation of the cohesive zone, and its extension becomes proportional to the bond length.

Since $s_{f}$ is in general very small, in short stiffeners the plateau can be hardly recognized, although for clarity of representation it has been evidenced in the graph on the right hand side of figure 7. On the contrary, long stiffeners exhibit a well-marked apparent yielding. This finding is in agreement with the experimental results, qualitatively recalled in figures 5 .

Eventually, one reaches point $\mathrm{D}$, characterized by condition $\delta_{0}=s_{f}$.

iii) Strain softening branch (point E).

After passing point D, it is again necessary to switch the control variable to the relative slip $\delta_{l}$ of the free end $\xi=l$. Increasing this parameter, the situation is like that of Figure 7 , point E. For a given $\delta_{l}$, one can find the length $c$ of the cohesive zone from the condition that relative slip $\delta_{d}$ at $\xi=d \equiv l-c$ is equal to $s_{f}$.

At this stage, the $P-\delta_{0}$ graph can be found from conditions analogous to (3.8), (3.9) and (3.10). The result is as represented on the right-hand-side of Figure 7. 


\section{Analytical results and comparison with experimental tests}

Most of the experiments in the technical literature are strain driven tests, which are not able to capture any snap-back response. An exception is the experimental campaign recently performed in the laboratories of the University of Parma and recorded in Carrara et al. (2011), where a closed-loop tensometer was used to control the force $P$ applied at a FRP stringer glued to concrete specimens according to the output of LVDT transducers. Two different controls where used. At the beginning of the loading history, the control parameter was the relative sliding of the loaded end of the stringer; successively, as the measured load tended to diminish, the control was switched to the relative sliding of the opposite free end of the stringer. This procedure is in agreement with the findings from the model just presented in Sections 3.2 and 3.3 .

Concrete prism of $150 \times 90 \times 300 \mathrm{~mm}$ nominal size were reinforced by Carbon-Fiber-reinforced Polymer stringers, $30 \mathrm{~mm}$ wide and $1.3 \mathrm{~mm}$ thick, with five different lengths $(30,60,90,120$ and $150 \mathrm{~mm})$. The measured mechanical properties of the materials used in Carrara et al. (2011) are reported in Table 1, together with the values corresponding to other experimental campaigns (Yuan et al., 2004; Mazzotti et al., 2008) that, although not using a closed loop control, can serve here as a significant comparison. For the same tests, Table 2 shows the parameters that define the interface law according to Figure 4. Following the proposed equivalency outlined in Section 3.1, we set $\tau_{c}=\tau_{f} / 2$ and $q_{c}=\tau_{c} \cdot b_{s}$, being $b_{s}$ the width of the stringer.

Using the data from Carrara et al. (2011), the results obtainable with the cohesive model are analyzed for a bond length of $150 \mathrm{~mm}$. Figure 8 shows the normalized interfacial shear force distribution $q / q_{c}$ at various stages of loading. For various values of the applied load, a cohesive zone length $c$ is calculated. Observe that at the loaded end the shear distribution tends to the value of the maximum allowable stress $q_{c}$, i.e.,

$$
\lim _{\xi \rightarrow c} \frac{q(\xi)}{q_{c}}=1
$$

This is not surprising because the shear stress at the frontier between the cohesive and the perfectly bonded zones has to be continuous. In general, 
Table 1: Mechanical properties of materials used in experimental campaigns.

\begin{tabular}{ccccccc}
\hline & \multicolumn{3}{c}{ Concrete } & \multicolumn{3}{c}{ FRP } \\
\cline { 2 - 6 } Test & $\begin{array}{c}\text { Elastic Modulus } \\
E_{p} \\
{[\mathrm{MPa}]}\end{array}$ & $\begin{array}{c}\text { Thickness } \\
t_{p} \\
{[\mathrm{~mm}]}\end{array}$ & $\begin{array}{c}\text { Width } \\
b_{p} \\
{[\mathrm{~mm}]}\end{array}$ & $\begin{array}{c}\text { Elastic Modulus } \\
E_{s} \\
{[\mathrm{MPa}]}\end{array}$ & $\begin{array}{c}\text { Thickness } \\
t_{s} \\
{[\mathrm{~mm}]}\end{array}$ & $\begin{array}{c}\text { Width } \\
b_{s} \\
{[\mathrm{~mm}]}\end{array}$ \\
\hline Carrara et al. (2011) & 28700 & 90 & 150 & 168500 & 1.3 & 50 \\
Yuan et al. (2004) & 28600 & 150 & 150 & 256000 & 0.165 & 25 \\
Mazzotti et al. (2008) & 30700 & 200 & 150 & 195200 & 1.2 & 50 \\
\hline
\end{tabular}

Note: When the literature provides only the cylindrical strength $f_{c k}$ then, as suggested in Italian structural Code (NTC 2008, 2008), $E_{p}$ has been calculated with $E_{p}=22000\left(f_{c m} / 10\right)^{0.3}$ $\mathrm{MPa}$, being $f_{c m}=f_{c k}+8 \mathrm{MPa}$.

Table 2: Parameters of interface law.

\begin{tabular}{lccc}
\hline Test & $\begin{array}{c}\tau_{f} \\
{[\mathrm{MPa}]}\end{array}$ & $\begin{array}{c}s_{1} \\
{[\mathrm{~mm}]}\end{array}$ & $\begin{array}{c}s_{f} \\
{[\mathrm{~mm}]}\end{array}$ \\
\hline Carrara et al. (2011) & 7.71 & 0.030 & 0.15 \\
Mazzotti et al. (2008) & 9.14 & 0.033 & 0.0971 \\
Yuan et al. (2004) & 7.20 & 0.034 & 0.16 \\
\hline
\end{tabular}

Note: When the literature does not provide the value for the peak stress $\tau_{f}$, then expressions of the Italian Code (CNR-DT/200, 2004) have been used, i.e., $\tau_{f}=$ $0.64 \kappa_{b} \sqrt{f_{c k} f_{c t m}}$ with $f_{c t m}=0.30 f_{c k}^{(2 / 3)}$ expressed in $\mathrm{MPa}$ and $\kappa_{b}=\sqrt{\frac{2-b_{s} / b_{p}}{1+b_{s} / 400[\mathrm{~mm}]}} \geq 1$. 
there is an approximation due to the finite number $n$ of terms used in the Chebyshev representation; the higher is $n$, the better is the approximation, and the graphs here correspond to the value $n=100$. At the other (free) edge of the stiffener, the solution still presents the singularity predicted by the theory of elasticity.

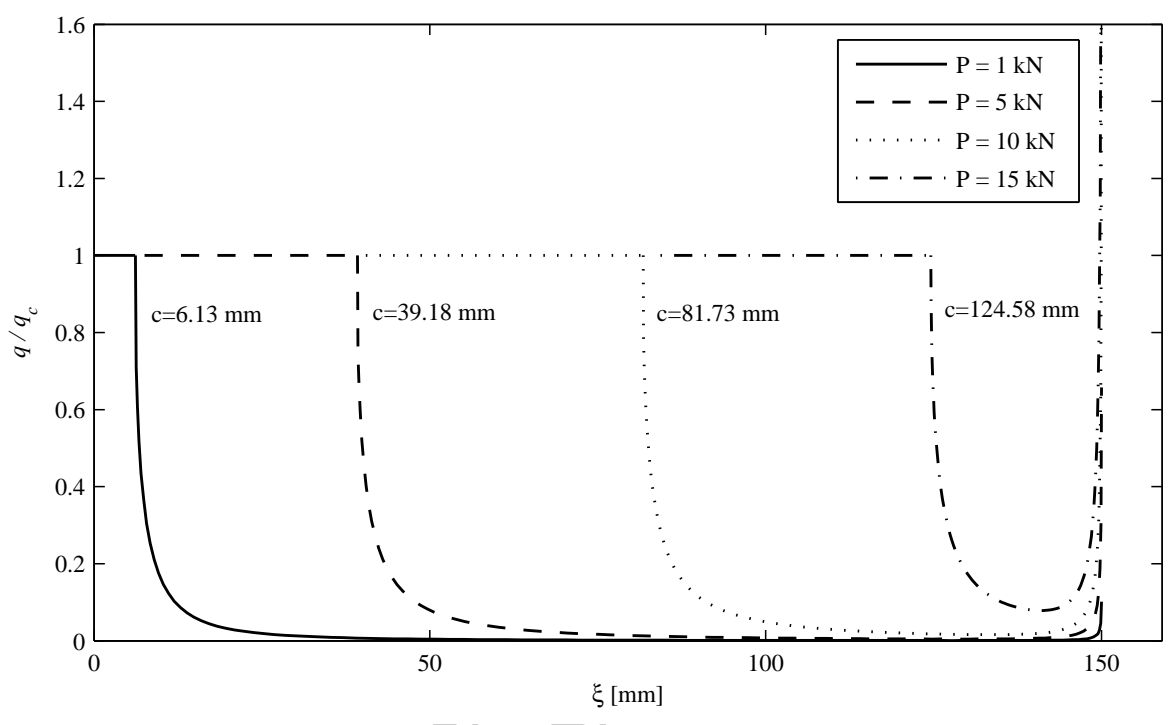

Figure 8: Interfacial shear force distribution for different values of the applied load and corresponding cohesive lengths $c$. Same materials of Carrara et al. (2011), with initial bond length $l=150 \mathrm{~mm}$.

Figure 9 represents the axial load $P$ as a function of the slip $\delta_{0}$ at the reference point $\xi=0$, calculated with no consideration of debonding, i.e., as if the interface had infinite ductility. Table 2 indicates that for the tests of Carrara et al. (2011) the failure slip is $s_{f}=0.15 \mathrm{~mm}$. Therefore when $\delta_{0}=0.15 \mathrm{~mm}$ debonding starts, and the corresponding load $P_{u}=15.09 \mathrm{kN}$ is the ultimate load. An effective bond length $c_{u}=125.4 \mathrm{~mm}$ corresponds to this case.

The example confirms that once the delamination begins and a progressive debonded length $d$ is developed, the cohesive length $c_{u}$ does not change considerably. The cohesive zone, once established, moves towards the free end of the stringer, while the applied loads remains substantially constant. 
A confirmation of this is in Figure 10, which represents the axial load $P$ as a function of the global slip $\delta_{d}$, now calculated at the end of the debonded zone $\xi=d$, for three different values $(d=0, d=5 \mathrm{~mm}, d=10 \mathrm{~mm})$ such that $d+c_{u}<l$. These cases correspond to the configurations $\mathrm{C}$ and $\mathrm{D}$ of Figure 6 . For each value of $d$, a new cohesive length is derived from condition (2.14) as a function of $P$. The three graphs in practice overlap, meaning that the response is substantially similar in all the cases when the bond length is greater than $c_{u}$. In particular, the value of the cohesive length when $\delta_{d}=s_{f}$ is independent of $d\left(c_{u}\right.$ varies in the range $\left.125.20 \div 125.41\right)$, while the critical load $P_{u}$ is practically constant $\left(P_{u} \simeq 15 \mathrm{kN}\right)$.

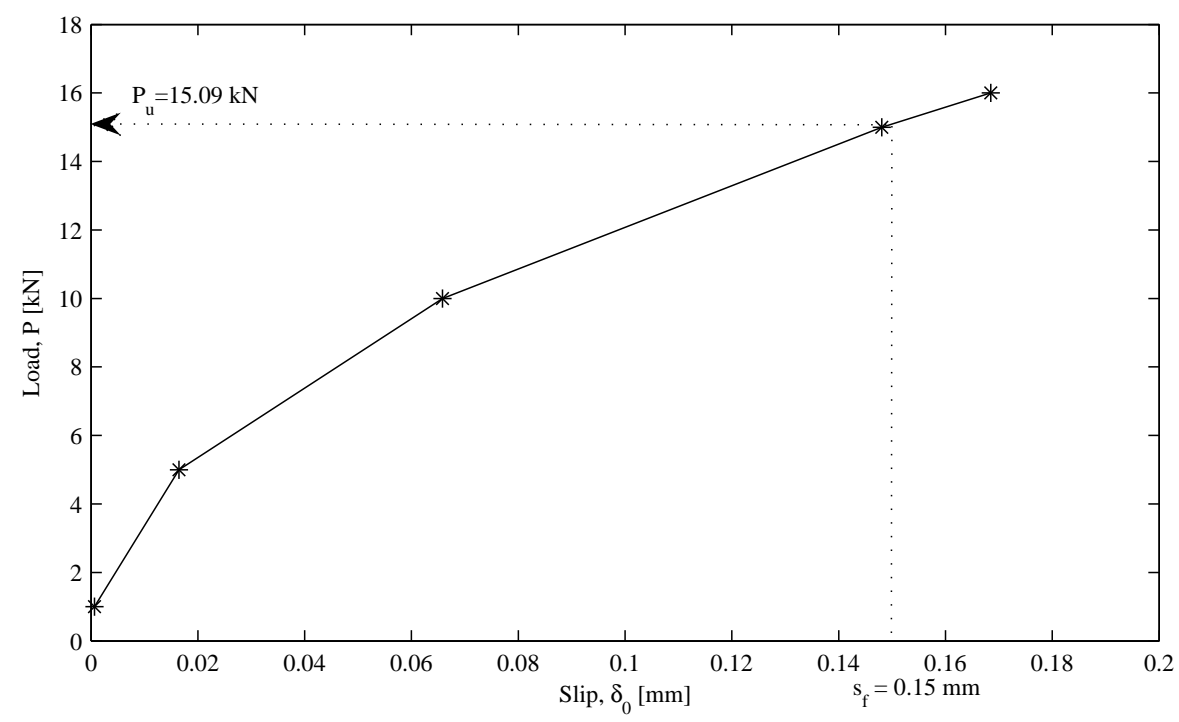

Figure 9: Load-slip $\left(P-\delta_{0}\right)$ curve for the same material parameters of Carrara et al. (2011) (Bond length $l=150 \mathrm{~mm}$ ).

In general, the length of the cohesive zone $c$ depends upon the value of the applied load $P$, independently of the bond length of the stiffener. This is also confirmed by Figure 11, which represents the value of $c$ associated with various values of the load $P$ for increasing values of the bond length l. Remarkably, $c$ does not substantially change as $l$ is varied, However, a minimum value of the bond length $l$ has to be associated to each load $P$. This derives from the condition that $l>P / q_{c}$, so that for a given value of load there is a minimum length necessary to develop the cohesive zone. 


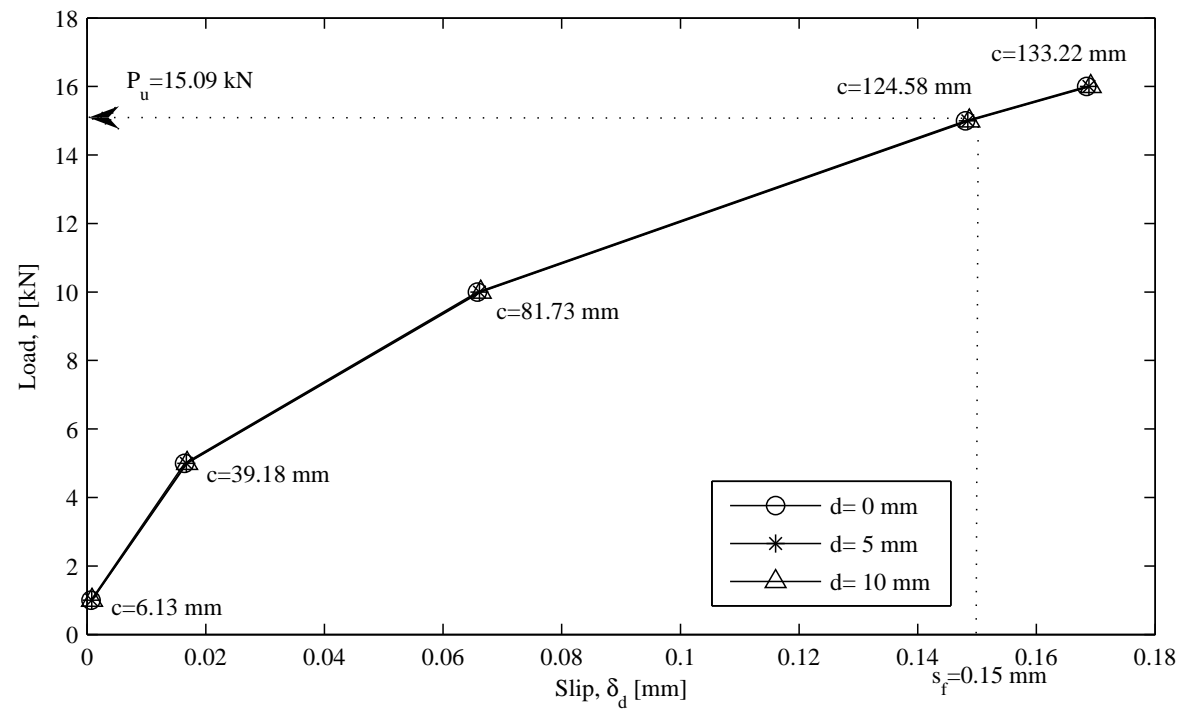

Figure 10: Load-slip curves $\left(P-\delta_{d}\right)$ for different values of the debonded length $d$ (Experimental data of Carrara et al. (2011)).

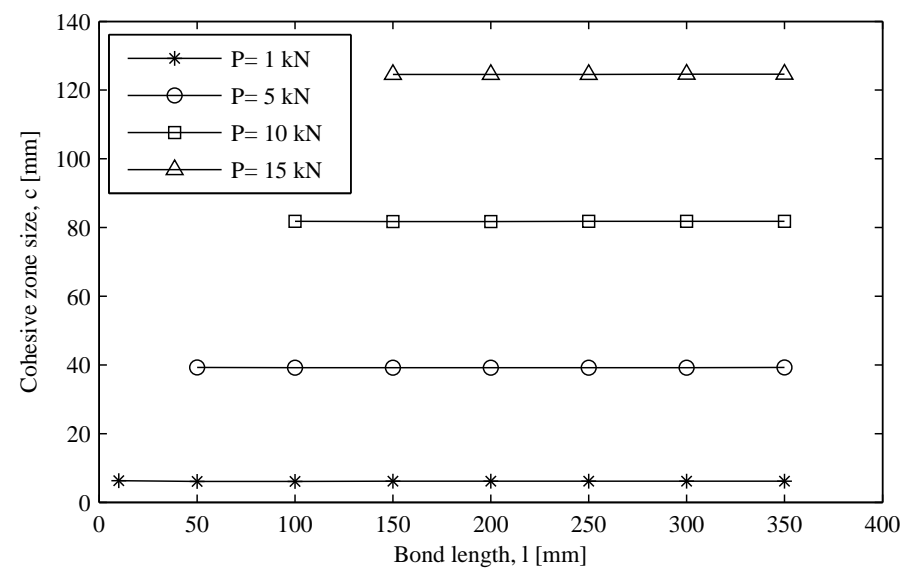

Figure 11: Length $c$ of the cohesive zone as a function of different bond lengths $l$ and axial loads $P$. 
It is interesting to investigate how the bond length $l$ affects the ultimate load $P_{u}$. Figures 12 show $P_{u}$ as a function of $l$, for the mechanical parameters reported in Tables 1 and 2 corresponding to the experimental works of (Yuan et al., 2004; Carrara et al., 2011; Mazzotti et al., 2008). Comparison with the experimental data, here indicated by dots, evidences that there is a good agreement with the prediction of the model for what the ultimate load is concerned. It is evident that $P_{u}$ increases with the bond length until a particular value is reached, after which the load remains almost constant. The value of the bond length that marks this transition is the effective bond length according to the classical definition, and matches very well with the value of $c_{u}$ calculated through the model, also reported in Figures 12. More precisely, all the results are summarized in Table 3, where in the "experimental data" columns, the mean experimental value on the peak load has been indicated with $P_{\max , e}$, while the approximate values of the bond length, evaluated from the change in the trend of the experimental data, have been referred to as $l_{e, e}$.

It may be useful to compare the results that derive from the model with those obtainable with formulas suggested by technical standards. To this respect, the recent Italian instructions CNR-DT200 (CNR-DT/200, 2004) appear to be one of the most modern reference. The main assumption in this model is a trilinear shear-stress vs. slip model, of the type represented in Figure 4, while the substrate is always considered rigid. The ultimate load is evaluated through an energetic balance leading to a formula of the type

$$
P_{\max }=\sqrt{2 E_{s} A_{s} G_{f} b_{s}}=b_{s} \sqrt{2 E_{s} t_{s} G_{f}},
$$

where $G_{f}$ is the fracture energy of the FRP-to-concrete interface. The effective bond length is obtained as

$$
l_{e}=\sqrt{\frac{E_{s} t_{s}}{2 f_{c t m}}},
$$

being $f_{c t m}$ the mean tensile strength of concrete (NTC 2008, 2008).

Using the data of Tables 1 and 2, the results are reported in Table 3, together with the prediction of the model just presented. Notice that the 
cohesive model, which is not based upon an energetic balance but simply relies upon the stress calculated with the classical theory of elasticity, gives values which are in excellent agreement with the standards for what the ultimate load is concerned. On the other hand, the expression (4.3) seems to excessively overestimate the bond length $l_{e}$ with respect to the experimental data, which are instead very well captured by our model.

Table 3: Results from cohesive zone model and comparison with the values predicted by Italian standards (CNR-DT/200, 2004) and experimental tests.

\begin{tabular}{ccccccc}
\hline & \multicolumn{2}{c}{ Cohesive Model } & Italian Standards & Experimental data \\
\cline { 2 - 6 } Test & $\begin{array}{c}P_{u} \\
{[\mathrm{kN}]}\end{array}$ & $\begin{array}{c}c_{u} \\
{[\mathrm{~mm}]}\end{array}$ & $\begin{array}{c}P_{\max } \\
{[\mathrm{kN}]}\end{array}$ & $\begin{array}{c}l_{e} \\
{[\mathrm{~mm}]}\end{array}$ & $\begin{array}{c}P_{\max , e} \\
{[\mathrm{kN}]}\end{array}$ & $\begin{array}{c}l_{e, e} \\
{[\mathrm{~mm}]}\end{array}$ \\
\hline Carrara et al. (2011) & 15.09 & 125.40 & 15.10 & 185.00 & 15.11 & $120 \div 150$ \\
Mazzotti et al. (2008) & 22.79 & 92.48 & 22.79 & 175.33 & 22.65 & $\sim 100.00$ \\
Yuan et al. (2004) & 5.49 & 60.05 & 5.51 & 91.89 & 5.53 & $\sim 60.00$ \\
\hline
\end{tabular}

It should be remarked that, as explained in Sections 3.2 and 3.3, if there is a part that remains completely bonded in a neighborhood of the free end of the stiffener, here elasticity theory predicts a singularity in stress. In a more accurate modeling, we have considered the possibility of a second cohesive zone at the free end of the stiffener, but we have verified that the length of this is much smaller (about $\frac{1}{1000}$ ) of the length of the cohesive zone at the loaded end. Consequently, the shear stress profile at the interface does not appreciably change if the singularity at the free end is removed. In other words, the part of the applied load that is equilibrated by the singularity is in general not important at all. In conclusion, the model just presented provides an accurate description of the debonding mechanism, at the price of a reasonable computational effort, which instead considerably increases when the second cohesive zone is introduced. 


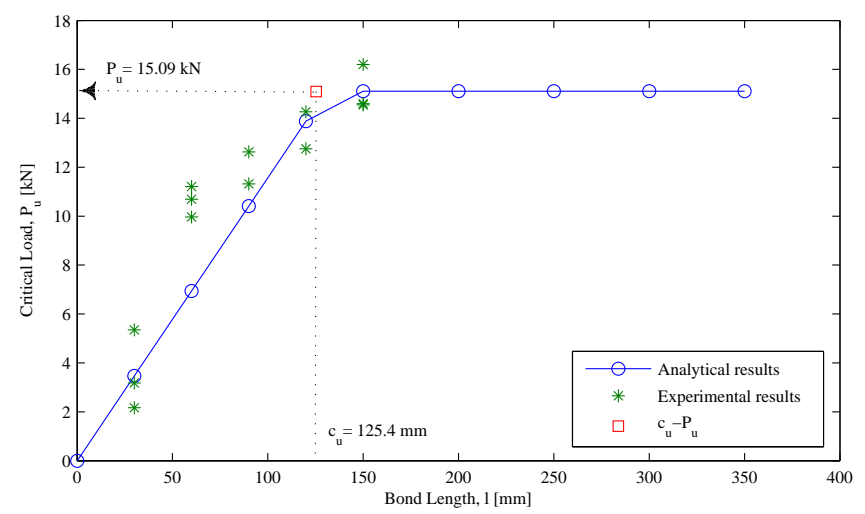

(a)

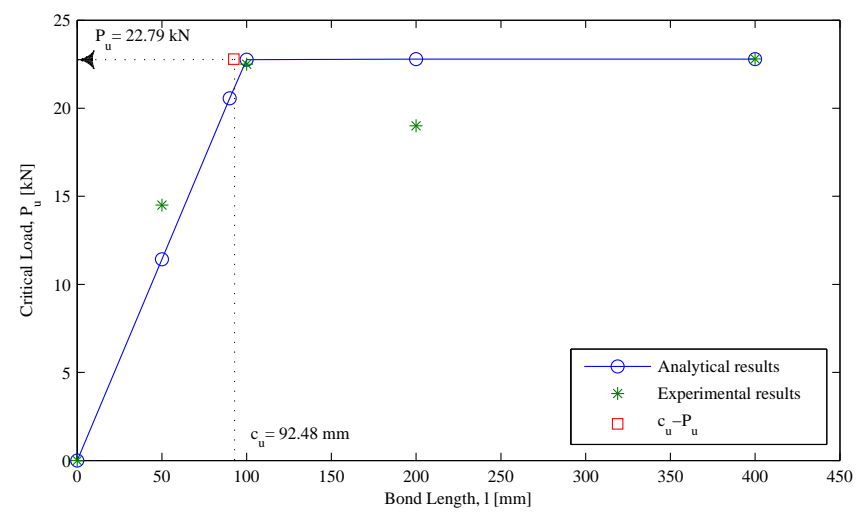

(b)

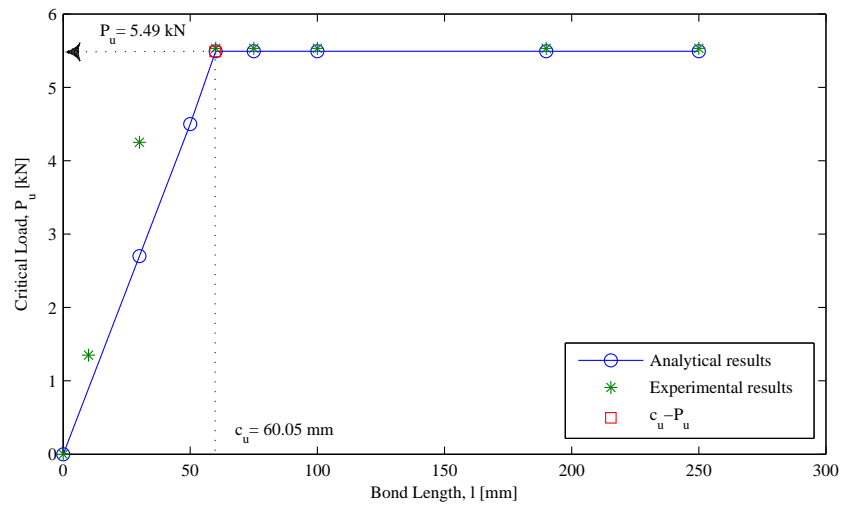

(c)

Figure 12: Ultimate load $P_{u}$ as a function of the initial bond length $l$. (a) Tests of Carrara et al. (2011); (b) Tests of Mazzotti et al. (2008); (c) Tests of Yuan et al. (2004). 


\section{Discussion and Conclusions}

An analytical model has been presented for the description of the interfacial debonding process in pure mode $I I$ of an elastic stiffener from a substrate. A practical motivation for this is the popular technique of strengthening concrete or masonry with stringers made of Fiber Reinforced Polymers (FRP). The contact problem has been analyzed under the hypothesis that the substrate is a linear elastic semi-infinite plate in generalized plane stress. Following the typical approach à la Barenblatt (Barenblatt, 1962), a shear type cohesive zone has been introduced in order to remove the singularity of the stress at the edge of the stiffener, imposing that the mode $I I$ stress intensity factor $K_{I I}$ is zero at the edge of the zone that remains completely bonded. Compatibility conditions for the relative displacement allow to obtain an integro-differential equation in terms of the tangential stresses (Grigolyuk and Tolkachev, 1987). The common technique of expansion in orthogonal polynomials is then used to obtain a set of algebraic equations, depending upon the unknown cohesive zone length $c$, whose value is determined with a root finding algorithm once the maximum allowable interface stress is given.

The major novelty of this approach is consideration of the elastic deformation of the substrate, which instead is considered rigid in all the models that we know dealing with FRP reinforcements. Indeed, if the substrate is rigid, the whole essence of the phenomenon must be captured through a shear stress $\tau$ vs. slip $s$ interface law between the materials in contact, but this simplification may give some inconsistency. For example, slip should occur through the whole bond, regardless of its length and of the level of the pull out load; perfect adhesion (no slip) can never be attained because it would give rise to infinite stress that no bonding could ever withstand.

On the other hand the proposed approach, just assuming a very simple stepwise $\tau-s$ interface law, naturally predicts the formation of a cohesive zone that annihilates the stress singularity in that part where perfect bonding is preserved. The cohesive length $c$ depends upon the maximum allowable cohesive shear-force $q_{c}$ at the interface between stringer and substrate and it results to be a function of the applied load. Remarkably, $c$ does not appreciably change if the bond length is increased.

If the stringer is sufficiently long, there is a maximal length $c_{u}$ of the cohesive zone. This is attained when the slip at the loaded end of the stiffener 
reaches the fracture slip $s_{f}$, representing a characteristic parameter of the interface constitutive model. Debonding initiates at this stage at a critical value $P_{u}$ of the load. The length $c_{u}$ does not change as delamination propagates along the interface, but simply translates as the debonding increases, until it reaches the opposite free end of the stringer. Therefore, $c_{u}$ depends upon the mechanical properties of the materials in contact, but not on the bond length $l$ of the stringer (of course, provided $l>c_{u}$ ). During this process the load $P$ remains almost constant and equal to $P_{u}$ because the majority by far of the applied load is equilibrated by the cohesive forces acting in the cohesive zone. Therefore, the length $c_{u}$ can be identified as the effective bond length, that is, the length that is necessary to assure the transfer of the load from the stiffener to the substrate; increase of the bond length beyond its effective value does not increse the load bearing capacity. This definition solves some ambiguities that are usually associated with the rigid-substrate hypothesis.

In general, the ultimate load $P_{u}$ is independent of the bond length $l$, provided that this is higher than the effective bond length $c_{u}$. When $l<$ $c_{u}$, the cohesive zone invades the whole bond length and the load bearing capacity of the joint is the product of $l$ and the maximum allowable cohesive shear-force $q_{c}$. Therefore, a basic distinction has to be made between long stringers, characterized by $l>c_{u}$ and short stringers, for which $l<c_{u}$. Long stringers exhibit a first strain-hardening branch in the load vs. slip graph, followed by a plateau associated with stable debonding (translation of the cohesive zone). The higher the bond length, the more marked is the plateau and, consequently, the higher the ductility of the joint. When the cohesive zone reaches the free end, a strain softening phase begins, usually characterized by a snap-back caused by the release of strain energy stored by the detached part of the FRP plate. Short stiffeners present a strain hardening branch, but the plateau phase is almost negligible, because there is no translation of the cohesive zone. In fact, the extension of the plateau, which is associated with a rigid translation of a fully yielded joint, can never exceed the fracture slip $s_{f}$. Obviously, the ultimate load and the apparent ductility for short stiffeners are always less than for long stiffeners.

The ultimate loads obtained through the model matches with representative experimental results present in the literature, and with the relevant formulas proposed in technical standards. For what the effective bond length 
is concerned, our analytical prediction are in very good agreement with experiments (at least with those that have been considered here). On the other hand, we must observe that the corresponding formulas suggested by technical standards give excessively overestimated values (about twice) with respect to test results. To this respect, our formulation seems to be an improvement of what proposed so far.

The model can be considered minimal, because it considers linear elastic materials and the simplest stepwise shear-stress vs. slip law for the interface. The latter assumption predicts a strain-softening snap-back branch which is parabolic in type, whereas the experimental results usually present a linear trend. Consideration of more elaborated interface laws could improve the performance of the model, but we may infer, albeit tentatively, that the formulation just presented is able to capture the essence of the debonding phenomenon before the snap-back phase occurs, the maximum load and the extension of the effective bond length. A numerical implementation of the model that uses the trilinear interface law commonly accepted in the technical literature will be the subject of further work.

Acknowledgements. The authors acknowledge for partial support the Italian government under the ReLUIS-DPC 2010-2013 research project and the European Community under contract) RFSR-CT-2012-00026 (S+G RFS-PR11017).

\section{Appendix A. Appendix: Chebyshev Polynomials}

The Chebyshev polynomials are usually defined introducing the variables

$$
t=\cos (\varphi), \varphi=\arccos (t) .
$$

The polynomials of the first kind take the form (Abramowitz and Stegun, 1964)

$$
T_{s}(t)=\cos (s \varphi(t))=\cos (s \arccos (t)),
$$

while the polynomials of the second kind are defined as 


$$
U_{s}(t)=\frac{\sin (s+1) \varphi(t)}{\sin (\varphi(t))}
$$

Both $T_{s}$ and $U_{s}$ form a sequence of orthogonal polynomials. The polynomials of the first kind are orthogonal with respect to the weight $1 / \sqrt{1-t^{2}}$ on the interval $[-1,1]$, that is,

$$
\int_{-1}^{1} \frac{T_{s}(t) T_{m}(t)}{\sqrt{1-t^{2}}} d t= \begin{cases}0, & \text { for } m \neq s \\ \frac{\pi}{2}, & \text { for } m=s \neq 0 \\ \pi, & \text { for } m=s=0\end{cases}
$$

Similarly, the polynomials of the second kind are orthogonal with respect to the weight $\sqrt{1-t^{2}}$ on the interval $[-1,1]$,i.e.,

$$
\int_{-1}^{1} U_{s}(t) U_{m}(t) \sqrt{1-t^{2}} d t= \begin{cases}0, & \text { for } m \neq s \\ \frac{\pi}{2}, & \text { for } m=s\end{cases}
$$

The following properties are useful:

$$
\int_{-1}^{1} \frac{T_{s}(t)}{\sqrt{1-t^{2}}\left(t-t_{0}\right)} d t= \begin{cases}0, & \text { for } s=0 \text { and }\left|t_{0}\right|<1 \\ \pi U_{s-1}\left(t_{0}\right), & \text { for } s>0 \text { and }\left|t_{0}\right|<1 \\ -\pi \frac{\left(t_{0}-\frac{\left|t_{0}\right|}{t_{0}} \sqrt{t_{0}^{2}-1}\right)^{s}}{\frac{\left|t_{0}\right|}{t_{0}} \sqrt{t_{0}^{2}-1}}, & \text { for } s \geq 0 \text { and }\left|t_{0}\right|>1\end{cases}
$$




$$
\begin{aligned}
\int_{-1}^{1} U_{s-1}(t) \sqrt{1-t^{2}} \ln \left|t-t_{0}\right| d t & \text { for } s=1 \text { and }\left|t_{0}\right|<1, \\
= & \begin{cases}-\frac{\pi}{2}\left(t_{0}^{2}+\ln 2\right), & \text { for } s>1 \text { and }\left|t_{0}\right|<1, \\
\frac{\pi}{2}\left[\frac{T_{s+1}\left(t_{0}\right)}{s+1}-\frac{T_{s-1}\left(t_{0}\right)}{s-1}\right], & \text { for } s=1 \text { and }\left|t_{0}\right|(\mathrm{A} . \bar{T}) \\
\frac{\pi}{4}\left[\left(\sqrt{t_{0}^{2}}-\sqrt{t_{0}^{2}-1}\right)^{2}+2 \ln \left|\frac{t_{0}+\frac{\left|t_{0}\right|}{t_{0}} \sqrt{t_{0}^{2}-1}}{2}\right|\right], & \text { for } s>1 \text { and }\left|t_{0}\right|>1 . \\
\frac{\pi}{2}\left(-\frac{\left|t_{0}\right|}{t_{0}}\right)^{s-1}\left[\frac{\left(\sqrt{t_{0}^{2}-1}-\sqrt{t_{0}^{2}}\right)^{s+1}}{s+1}-\frac{\left(\sqrt{t_{0}^{2}-1}-\sqrt{t_{0}^{2}}\right)^{s-1}}{s-1}\right], & \end{cases}
\end{aligned}
$$

Another property of the Chebyshev polynomials is that, in the interval $-1 \leq t \leq 1$, they attain the maximum and minimum values at the endpoints, given by

$$
\left\{\begin{array}{l}
T_{s}(1)=1 \\
T_{s}(-1)=(-1)^{s} \\
U_{s}(1)=s+1 \\
U_{s}(-1)=(s+1)(-1)^{s}
\end{array}\right.
$$

These relationships are of help while estimating qualitative properties of the solution.

\section{References}

Abramowitz, M., Stegun, I., 1964. Handbook of Mathematical Functions: With Formulas, Graphs, and Mathematical Tables. Applied Mathematics Series, Dover Publications.

Ali-Ahmad, M., Subramaniam, K., Ghosn, M., 2006. Experimental investigation and fracture analysis of debonding between concrete and FRP sheets. J. Eng. Mech. 132, 914-923.

Arutiunian, N., 1968. Contact problem for a half-plane with elastic reinforcement. J. Appl. Math. Mech. 32, 652-665. 
Ascione, F., Mancusi, G., 2010. Axial/bending coupled analysis for FRP adhesive lap joints. Mech. Adv. Mater. Struct. 17, 85-98.

Ascione, F., Mancusi, G., 2012. Curve adhesive joints. Compos. Struct. 94, $2657-2664$.

Barenblatt, G., 1962. The mathematical theory of equilibrium cracks in brittle fracture, Elsevier. volume 7 of Advances in Applied Mechanics, pp. $55-129$.

Begley, M.R., Collino, R., Israelachvili, J.N., McMeeking, R.M., 2012. Peeling of a tape with large deformations and frictional sliding. J. Mech. Phys. Solids In press.

Benscoter, S., 1949. Analysis of a single stiffener on an infinite sheet. J. Appl. Mech. 16, 242-246.

Bizindavyi, L., Neale, K., 1999. Transfer lengths and bond strengths for composites bonded to concrete. J. Compos. Constr. 3, 153-160.

Brosens, K., Van Gemert, D., 1997. Anchoring stresses between concrete and carbon fibre reinforced laminates. Proc. 3rd Int. Symp. Non Metallic (FRP) Reinf. Concr. Struct. 1, 271-278.

Brown, E.H., 1957. The diffusion of load from a stiffener into an infinite elastic sheet. Proc. R. Soc. London, Ser. A 239, 296-310.

Carloni, C., Subramaniam, K.V., 2010. Direct determination of cohesive stress transfer during debonding of FRP from concrete. Compos. Struct. 93, $184-192$.

Carloni, C., Subramaniam, K.V., Savoia, M., Mazzotti, C., 2012. Experimental determination of FRP - concrete cohesive interface properties under fatigue loading. Compos. Struct. 94, $1288-1296$.

Carrara, P., Ferretti, D., Freddi, F., Rosati, G., 2011. Shear tests of carbon fiber plates bonded to concrete with control of snap-back. Eng. Fract. Mech. 78, 2663 - 2678.

Chen, B., Wu, P., Gao, H., 2009. Geometry- and velocity-constrained cohesive zones and mixed-mode fracture/adhesion energy of interfaces with periodic cohesive interactions. Proc. R. Soc. A 465, 1043-1053. 
Chen, G., Teng, J., Chen, J., 2012. Process of debonding in rc beams shearstrengthened with FRP u-strips or side strips. Int. J. Solids Struct. 49, $1266-1282$.

Chen, J., Teng, J., 2001. Anchorage strength models for FRP and steel plates bonded to concrete. J. Struct. Eng. 127, 784-791.

CNR-DT/200, 2004. Guide for the design and construction of externally bonded FRP systems for strengthening exixting structures. National Research Council (CNR). [In Italian].

Dal Corso, F., Bigoni, D., Gei, M., 2008. The stress concentration near a rigid line inclusion in a prestressed, elastic material. part I: Full-field solution and asymptotics. J. Mech. Phys. Solids 56, 815-838.

De Faveri, S., Freddi, L., Paroni, R., 2012. No-tension bodies: a reinforcement problem. Sumbmitted to Europ. J. Mech.

De Lorenzis, L., Miller, B., Nanni, A., 2001. Bond of fiber-reinforced polymer laminates to concrete. ACI Mat. J. 98, 256-264.

Ferracuti, B., Savoia, M., Mazzotti, C., 2007. Interface law for FRPconcrete delamination. Compos. Struct. 80, $523-531$.

Franco, A., Royer-Carfagni, G., 2012. Energetic balance in the debonding of a reinforcing stringer: Effect of the substrate elasticity. Sumbmitted to Int. J. Solids Struct.

Freund, L., Suresh, S., 2008. Thin Film Materials: Stress, Defect Formation and Surface Evolution. Cambridge University Press.

Grigolyuk, E., Tolkachev, V., 1987. Contact Problems in the Theory of Plates and Shells. Moscow: Mir Pub.

Holzenkämpfer, P., 1994. Ingenieurmodelle des Verbunds geklebter Bewehrung für Betonbauteile, Dissertation. Ingenieurmodell für das Verbundsystem Stahlbeton + Klebelasche / Institut für Baustoffe, Massivbau und Brandschutz Braunschweig, IBMB, TU.

Hutchinson, J., Suo, Z., 1991. Mixed mode cracking in layered materials, Elsevier. volume 29 of Advances in Applied Mechanics, pp. 63-191. 
Kamel, A., Elwi, A., Cheng, R., 2004. Experimental investigation on FRP sheets bonded to concrete. Emirates J. Eng. Res. 9, 71-76.

Koiter, W.T., 1955. On the diffusion of load from a stiffener into a sheet. Quart. J. Mech. Appl. Math. 8, 164-178.

Maeda, T., Asano, Y., Sato, Y., Ueda, T., Kakuta, Y., 1997. A study on bond mechanism of carbon fiber sheet. Proc. 3rd Int. Symp. Non Metallic (FRP) Reinf. Concr. Struct. 1, 279-285.

Mancusi, G., Ascione, F., 2012. Performance at collapse of adhesive bonding. Compos. Struct. In press.

Mazzotti, C., Savoia, M., Ferracuti, B., 2008. An experimental study on delamination of FRP plates bonded to concrete. Constr. Build. Mater. 22, $1409-1421$.

Melan, E., 1932. Ein beitrag zur theori geschweisster verbindungen. Ing. Arch. 3, 123-129.

Neubauer, U., Rostasy, F., 1997. Design aspects of concrete structures strengthened with externally bonded CFRP plates. Proc. 7th Int. Conf. on Struct. Faults and Repair 2, 109-118.

NTC 2008, 2008. New Technical standards for Construction. Italian Ministry of Public Works (DM 14.01.08). [In Italian].

Reissner, E., 1940. Note on the problem of the distribution of stress in a thin stiffened elastic sheet. Proc. Natl. Acad. Sci. U.S.A. 26, 300-305.

Shield, T., Kim, K., 1992. Beam theory models for thin film segments cohesively bonded to an elastic half space. Int. J. Solids Struct. 29, 1085 1103.

Subramaniam, K.V., Carloni, C., Nobile, L., 2007. Width effect in the interface fracture during shear debonding of FRP sheets from concrete. Eng. Fract. Mech. 74, $578-594$.

Taljsten, B., 1997. Defining anchor lengths of steel and CFRP plates bonded to concrete. Int. J. Adhes. Adhes. 17, 319 - 327. 
Williams, J., Hadavinia, H., 2002. Analytical solutions for cohesive zone models. J. Mech. Phys. Solids 50, $809-825$.

Wu, Z., Yuan, H., Niu, H., 2002. Stress transfer and fracture propagation in different kinds of adhesive joints. J. Eng. Mech. 128, 562-573.

Yao, J., Teng, J., Chen, J., 2005. Experimental study on FRP-to-concrete bonded joints. Comp. B: Eng. 36, $99-113$.

Yuan, H., Teng, J., Seracino, R., Wu, Z., Yao, J., 2004. Full-range behavior of FRP-to-concrete bonded joints. Eng. Struct. 26, 553-565.

Yuan, H., Wu, Z., Yoshizawa, H., 2001. Theoretical solutions on interfacial stress transfer of externally bonded steel/composite laminates. J. Struct. Mech. Earth. Eng. 18, 27-39. 